\title{
Turbulent Flow in Shock Tubes of Varying Cross Section*
}

\author{
Robert F. Dressler
}

\begin{abstract}
The unsteady escape flow of a compressible gas is investigated subject to the influences of varying duct cross section and mechanical retardation due to turbulence and frictional dissipation. This study is applicabıe to shock tubes, intermittent wind tunnels, and general pneumatic-control systems, such as airbrakes. Wave-front velocities and trajectories are obtained by direct integration along the bounding Mach lines. In the general case, calculation of first-order approximations is reduced to a set of independent ordinary differential equations for easy stepwise integration. For the special cases of constant resistance or exponentially converging-diverging ducts, first-order solutions are obtained analytically. An error estimate is derived. Local Mach number variations are presented throughout the flow.
\end{abstract}

\section{Introduction}

One type of wind tunnel in present use employs the principle of intermittent discharge of atmospheric air into a vacuum chamber, or intermittent high-pressure discharge into an atmospheric chamber. In the latter case, a rapidly advancing shock discontinuity will also be generated in the forward wave. The familiar Riemann shock tube furnishes such an example; for this type of duct Riemann $[1]^{1}$ studied the basic theory of one-dimensional unsteady gas flows. In the present paper the emphasis will be upon the flow immediately after the diaphragm is broken, not upon the flow developing after a sufficiently long time when an almost steady-state regime is approached. The intermittent-vacuum-type system gives a more constant stagnation temperature and requires less power than the intermittent-pressure type, but gives lower densities in the test section. In the first stages of operation, these flows in shock tubes or vacuum-type wind tunnels can be approximately described by the known centered simple-wave solution [2, ch. III], defining a subsonic-supersonic pattern about the diaphragm position. It is the purpose of this paper to investigate quantitatively the possible improvement in density, velocity, or Mach number that might be created if such an unsteady flow is made to pass through a test section of varying cross section; at the same time the retarding effect due to wall resistance and ensuing turbulence will also be considered.

Any forward shock discontinuity that would be created in an actual flow if it propagates into a region containing some gas will be ignored in the present treatment. Even if a relatively strong shock exists, the results to be derived here will still be approximately applicable to the backward moving rarefaction wave region. This will furnish quantitative information, for example, concerning frictional effects in the rarefaction waves propagating through pneumaticcontrol circuits, such as airbrake lines.

The case of steady flows in variable ducts has recently been studied by Meyer [3], emphasizing the propagation of advancing and receding wave fronts due to a finite disturbance superimposed upon a basic steady flow, with consideration given to the varying cross-section effect, but not the resistance effect.

Dimensional quantities (barred) will first be introduced to define the dimensionless ones (unbarred), which will then be used throughout this paper. In the standard notation as given in [2], $\bar{x}$ is taken in the direction of flow; time is denoted by $\bar{t}$, with the valve or diaphragm at $\bar{x}=0$ opened at $\bar{t}=0$. Let $\bar{p}$ denote pressure, $\bar{\rho}$ the mass density, and $\bar{u}$ the velocity component in the downstream direction. We consider the nonlinear flow of a polytropic gas having the equation of state

$$
\bar{p}=\bar{A} \bar{\rho}^{\gamma},
$$

*The preparation of this paper was sponsored by the Office of Naval Research, USN

1 Figures in brackets indicate the literature references at the end of this paper. 


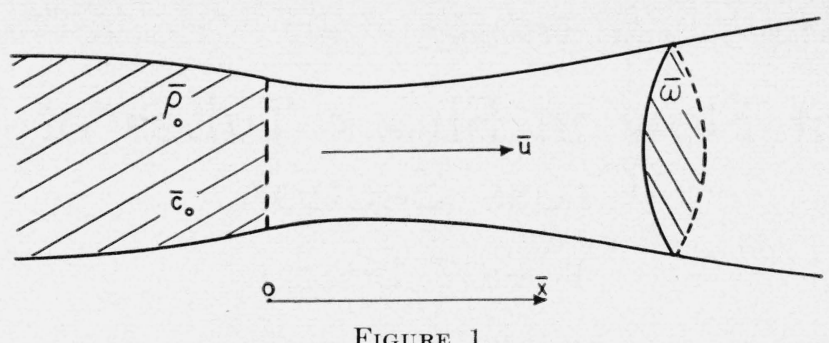

FIGURE 1 .

with known constant $\bar{A}$ and adiabatic exponent $\gamma$. We introduce in the usual manner the local sound speed $\bar{c}$ into the differentiated equation of state,

$$
\bar{c}^{2}=\gamma \bar{A} \bar{\rho}^{\gamma-1} .
$$

For $\bar{t}<0$, the gas is at rest to the left of the valve, $\bar{x}<0$, and has properties $\bar{\rho}_{0}, \bar{c}_{0}$, and $\bar{u}_{0}=0$. For $\bar{t}<0$, there is no gas in $\bar{x}>0$ (see fig. 1). We assume the flow to occur in a duct with cross section varying gradually enough to keep the flow essentially one-dimensional with uniform velocity over any cross section, and subject to the retarding force from the wall friction and turbulence, depending empirically upon the square of the velocity and the roughness coefficient for the wall surface. The dynamic equation is then

$$
\frac{\partial \bar{u}}{\partial \bar{t}}+\bar{u} \frac{\partial \bar{u}}{\partial \bar{x}}+\frac{2}{\gamma-1} \bar{c} \frac{\partial \bar{c}}{\partial \bar{x}}+\epsilon \bar{R}(\bar{x}) \bar{u}^{2}=0
$$

where $\epsilon$ is a small dimensionless expansion parameter, and the given function $\bar{R}(\bar{x})>0$ varies if the roughness of the wall surface changes from region to region, or if the cross section varies, thus varying the hydraulic radius ${ }^{2}$ of the duct in a known manner. Letting $\bar{\omega}(\bar{x})$ denote the

area of the cross section of the duct, the modified equation of continuity will be

$$
2 \frac{\partial \bar{c}}{\partial \bar{t}}+2 \bar{u} \frac{\partial \bar{c}}{\partial \bar{x}}+(\gamma-1) \bar{c} \frac{\partial \bar{u}}{\partial \bar{x}}+(\gamma-1) \epsilon \bar{a}(\bar{x}) \bar{u} \bar{c}=0,
$$

in which $\bar{a}(\bar{x})=[d \bar{\omega} / d \bar{x}] / \bar{\omega}$, with $\epsilon \bar{a}=0$ corresponding to a uniform duct.

In eq (1), (3), and (4) we have tacitly assumed constant entropy for the flow. This is a simplification tantamount to neglecting the heat change (and hence its extra resulting effects upon the mechanics of the gas flow) which will always be produced by the operation of the resistance term in converting kinetic energy into thermal energy. The present discussion will consider the mechanics of the flow including only the retarding force, not the full thermodynamical aspects of the problem. To do so would require also the inclusion of the energy equation, creating a system of three equations in the three unknowns: particle velocity, sound velocity, and specific entropy. Such a system has an associated set of six characteristic equations; these have been used by Hall [4] and others for purposes of quite arduous numerical integrations performed over characteristic meshes in the $(x, t)$ plane. The simplified model used in our present paper can be described also in the following manner. We consider a onedimensional flow through a tube with the mass traveling lin thin slices perpendicular to the walls. Each slice is subjected to a retarding shear force distributed over its peripheral area. This force $F(\rho, u)=-\lambda \rho u^{2}$ varies with the changing density and velocity of the moving slice. Results obtained for this model will thus extract the purely mechanical effect of the friction from the full nonisentropic thermodynamical effects.

${ }^{2}$ If $\bar{k}(\bar{x})$ measures the roughness of the wall material, then $\bar{R}=\bar{k}(\bar{x}) / \bar{T}(\bar{x})$, where the hydraulic radius $\bar{r}$ is defined as $\bar{\omega} / \bar{P}$. $\bar{P}$ is the perimeter of the cross section having area 
Now introduce the unbarred dimensionless quantities defined by $x=\left(g / \bar{c}_{0}^{2}\right) \bar{x}, \bar{t}=\left(g / \bar{c}_{0}\right) \bar{t}$, $u=\left(1 / \bar{c}_{0}\right) \bar{u}, c=\left(1 / \bar{c}_{0}\right) \bar{c}, \rho=\left(1 / \bar{\rho}_{0}\right) \bar{\rho}, R=\left(\bar{c}_{0}^{2} / g\right) \bar{R}, a=\left(\bar{c}_{0}^{2} / g\right) \bar{a}$, and abbreviate $2 /(\gamma-1)=l$, the unmodified escape speed. Equations (3) and (4) then become

$$
\begin{aligned}
& \frac{\partial u}{\partial t}+u \frac{\partial u}{\partial x}+l c \frac{\partial c}{\partial x}+\epsilon R(x) u^{2}=0 \\
& l \frac{\partial c}{\partial t}+l u \frac{\partial c}{\partial x}+c \frac{\partial u}{\partial x}+\epsilon a(x) u c=0
\end{aligned}
$$

The escape flow solution for $\epsilon=0$ (uniform duct and resistance neglected) is

$$
\begin{aligned}
u^{0} & =\frac{2}{\gamma+1}(1+m) \\
c^{0} & =\frac{\gamma-1}{\gamma+1}(l-m)
\end{aligned}
$$

where $m=x / t$. This is supersonic in the forward region $0<x \leq l t$; subsonic in the backwave $-t \leq x<0$; and $u^{0}=c^{0}=2 /(\gamma+1)$ at the fixed sonic point $x=0$.

The equations for the Mach lines of the system (5), (6) are

$$
\begin{aligned}
& I_{+}: \frac{d x}{d t}=u-c \\
& I_{-}: \frac{d x}{d t}=u+c \\
& I I_{+}: d(u-l c)=-\epsilon(R u-a c) u d t \\
& I I_{-}: d(u+l c)=-\epsilon(R u+a c) u d t
\end{aligned}
$$

where the $I_{+}$and $I I_{+}$relations hold along the $b_{+}$family of receding Mach lines in the $(x, t)$ plane, and the other pair along the $b_{-}$advancing lines. The Mach lines $\mathrm{OB}$ and $\mathrm{OF}$ in figure 2 define the moving limits of the flow zone. As the quantities $R(x)$ and $a(x)$ do not appear in the first equation of (9), it follows that the curve OB must remain a straight line with direction $m=-1$ in all cases.

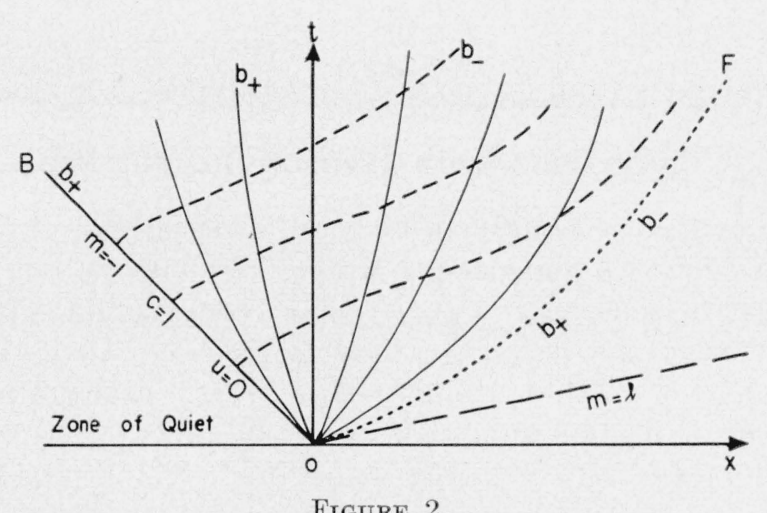

FIGURE 2. 


\section{Locus of the Wavefront}

It is seen at once from the last two equations in (9) that, within the accuracy of the basic relations (5) and (6), the duct shape has no effect upon the wavefront trajectory where $c=0$ because the terms containing $a(x)$ will vanish along this wave front. Its locus is a function of $\epsilon R$, however, and will remain a double Mach line as the plus and minus relations coincide for $c=0$ in (9). The $I$ and $I I$ pair of equations can then be integrated explicitly to obtain the exact wavefront locus, which is

$$
t_{F}(x)=\frac{\gamma-1}{2} \int_{0}^{x} e^{\int_{0}^{\zeta} \Re(\xi) d \xi} d \zeta
$$

in which $\epsilon$ is included in the notation $\epsilon R=\Re$. The velocity of the wavefront is given by

$$
u_{F}(x)=\frac{2}{\gamma-1} e^{-\int_{0}^{x} \Re(\xi) d \xi} .
$$

In particular, if $\Re=$ constant, e. g., in a duct having homogeneous wall surface and uniform cross section, the position and velocity will be

$$
\begin{aligned}
& x_{F}=\frac{1}{\Re} \ln \left(\frac{2 \Re}{\gamma-1} t+1\right) \\
& u_{F}=\left(\Re t+\frac{\gamma-1}{2}\right)^{-1} .
\end{aligned}
$$

These exact results will be used later to furnish an error estimate for the approximations to be derived for the interior wave zone. The forward wave velocity will not approach asymptotically any nonzero value, but will continually slow down, approaching, but never attaining, a state of rest. This is true more generally for any variable $R(x)$ with $a(x)$ arbitrary, as can be seen from (11), because $R(x)$ is always nonnegative.

The Mach lines inside the flow will be locally distorted by the influence of $R$ and $a$ from their positions for the basic solution (7) and (8), which are

$$
\begin{aligned}
& b_{+}: x=m t,-1 \leq m \leq 2 /(\gamma-1), \\
& b_{-}:\left(\frac{x}{p}\right)=\frac{2}{\gamma-1}\left(\frac{t}{p}\right)-\frac{\gamma+1}{\gamma-1}\left(\frac{t}{p}\right)^{\frac{3-\gamma}{1+\gamma}}, \quad 0<a<\infty,
\end{aligned}
$$

where each $b_{-}$curve emanates from the point $(-p, p)$ on $\mathrm{OB}$.

\section{Comparison with Hydrodynamic Problem}

If $\epsilon=0$ in (3) and (4), the equations become identical for $\gamma=2$ with the approximate Saint-Venant equations for two-dimensional water flow with a free surface, where $c^{2}$ is the water height, and $u$ is the horizontal velocity of a cross section. If this analogy extended also to the equations when the resistance term is included, then the present aerodynamic problem would be analogous to the hydraulic "dam-break" problem previously considered by the author in [5]. The hydraulic-resistance function, however, is more complicated in an essential way, taking the form $-R u^{2} / c^{2}$ rather than $-R u^{2}$. The hydraulic dam-break wavefront contains a second-order pole singularity, and its locus is thereby not a characteristic curve itself, but only an envelope of such curves. Explicit integration for its trajectory is apparently not possible in that case. The simpler nature of the present problem permits carrying the different mathematical results somewhat farther and with less approximation involved for the basic model used. 


\section{Functions for First-Order Effects}

Using (7) and (8), we substitute

$$
\begin{aligned}
& u(x, t, \boldsymbol{\epsilon})=u^{0}(x, t)+U(x, t) \boldsymbol{\epsilon}+O\left(\boldsymbol{\epsilon}^{2}\right) \\
& c(x, t, \boldsymbol{\epsilon})=c^{0}(x, t)+C(x, t) \boldsymbol{\epsilon}+O\left(\boldsymbol{\epsilon}^{2}\right)
\end{aligned}
$$

into (5) and (6). The terms in $\epsilon$ define the system

$$
\begin{gathered}
(\gamma+1) \frac{\partial U}{\partial t}+2\left(\frac{x}{t}+1\right) \frac{\partial U}{\partial x}+2\left(l-\frac{x}{t}\right) \frac{\partial C}{\partial x}+\frac{2}{t} U-\frac{2}{t} C+\frac{4}{\gamma+1}\left(\frac{x}{t}+1\right)^{2} R(x)=0 \\
(\gamma-1)\left(l-\frac{x}{t}\right) \frac{\partial U}{\partial x}+l(\gamma+1) \frac{\partial C}{\partial t}+2 l\left(\frac{x}{t}+1\right) \frac{\partial C}{\partial x}-\frac{2}{t} U+\frac{2}{t} C+2\left(\frac{\gamma-1}{\gamma+1}\right)\left(l-\frac{x}{t}\right)\left(\frac{x}{t}+1\right) a(x)=0
\end{gathered}
$$

This system for $U$ and $C$ has the same known Mach lines in the $(x, t)$ plane as deiined in (13) and (14), along which the following Riemann relations will hold:

$$
\begin{aligned}
& b_{+}: d(U-l C)=\left[\frac{4}{(\gamma+1) t}(C-U)-\left(R u^{0}-a c^{0}\right) u^{0}\right] d t \\
& b_{-}: d(U+l C)=-\left(R u^{0}+a c^{0}\right) u^{0} d t .
\end{aligned}
$$

This second equation is independent of $U$ and $C$ on the right side; therefore, it is possible for given $R(x)$ and $a(x)$ to integrate the line integral explicitly along each known $b_{-}$curve, using (14). The result will be a known expression of form

$$
U+l C=\Phi(t, p) .
$$

This will then permit a very easy separate step-wise numerical integration along each $b_{+}$ curve to solve for $U$ and $C$. Thus the problem is reduced to a set of independent "one-dimensional" numerical calculations for a single ordinary differential equation along each $b_{+}$curve. This is a calculation of much greater simplicity than the two-dimensional numerical procedure that would be necessary for the full problem in (5), (6), and (9).

\section{Integration in Special Cases}

Instead of using the numerical approach for arbitrary $R$ and $a$, we return to solve the basic eq (16) explicitly for the special case when $R$ and $a$ are both constants. In particular, $a=0$, $R=$ constant $>0$ will describe the resistive effects on the flow in a duct with any fixed cross section and homogeneous wall roughness. The combination $a=$ constant $\neq 0, R=0$ will describe the effect of a converging or diverging duct, neglecting resistance effects. For example, $a>0$ can describe a duct with circular cross section, exponentially diverging, or with rectangular cross section, constant width, also exponentially diverging; $a<0$ corresponds to exponentialiy converging ducts of either type. The combination $R$ and $a$, both nonzero constants, is less meaningful physically, because in such a varying duct the wall roughness would have to be varied in conformity with the changing hydraulic radius in order to maintain $R$ constant. Therefore, the contributions of $R$ and $a$ will be considered separately, although the solution will be made for both simultaneously.

First, we put $\gamma=1.4$ for air to shorten the expressions; the escape velocity is then $l=5$. 
System (16) has for its general solution

$$
\begin{aligned}
U(x, t)= & -\frac{5}{t} K\left(\frac{x}{t}\right)-\frac{25}{144}\left[84-36\left(5-\frac{x}{t}\right)+5\left(5-\frac{x}{t}\right)^{2}\right] R t-\frac{5}{48}\left[2\left(5-\frac{x}{t}\right)-\left(5-\frac{x}{t}\right)^{2}\right] a t+F(\alpha), \\
C(x, t)= & \frac{1}{t} K\left(\frac{x}{t}\right)-\frac{5}{144}\left[60-36\left(5-\frac{x}{t}\right)+7\left(5-\frac{x}{t}\right)^{2}\right] R t-\frac{1}{48}\left[10\left(5-\frac{x}{t}\right)-3\left(5-\frac{x}{t}\right)^{2}\right] a t+\frac{\delta}{\alpha}+ \\
& \frac{1}{5} F(\alpha)+\frac{4}{5 \alpha} \int^{\alpha} F(\xi) d \xi
\end{aligned}
$$

where $\alpha=(5-x / t) t^{1 / 3}, K$ and $F$ are arbitrary functions, and $\delta$ is an arbitrary constant. The form of this solution indicates that $U$ and $C$ must be either infinite or zero at $t=0$; hence we must choose $K=0$ and $\delta=0$ to keep the functions zero. The boundary conditions $U=C=0$ along line $\mathrm{OB}$ then determine the function $F=(1 / 1296)(50 R-15 a)(5-x / t)^{3} t$. With $x / t=m$, the main results reduce to

$$
\begin{aligned}
& U=-\frac{25}{1,296}\left(2 m^{3}+15 m^{2}+24 m+11\right) R t+\frac{5}{432}\left(m^{3}-6 m^{2}+3 m+10\right) a t \\
& C=-\frac{5}{1,296}\left(4 m^{3}+3 m^{2}-6 m-5\right) R t+\frac{1}{432}\left(2 m^{3}-3 m^{2}-30 m-25\right) a t
\end{aligned}
$$

Denoting $\epsilon R t=\sigma$ and $\epsilon a t=\tau$, the flow velocity and local sound speed correct to first order can be written from (19), using (7) and (8), in the form

$$
\begin{aligned}
& u=u^{\circ}+h_{1}(m) \sigma+h_{2}(m) \tau \\
& c=c^{\circ}+k_{1}(m) \sigma+k_{2}(m) \tau .
\end{aligned}
$$

Once $u$ and $c$ have been calculated by (19) and (20), the pressure and density are immediately known through relations (1) and (2).

\subsection{Effects on Velocity and Density}

To provide immediate perception of the various effects, the $h, k$ polynomials are graphed in figure 3 , in which $h_{1}$ is plotted to a separate vertical scale given on the right. We note that an

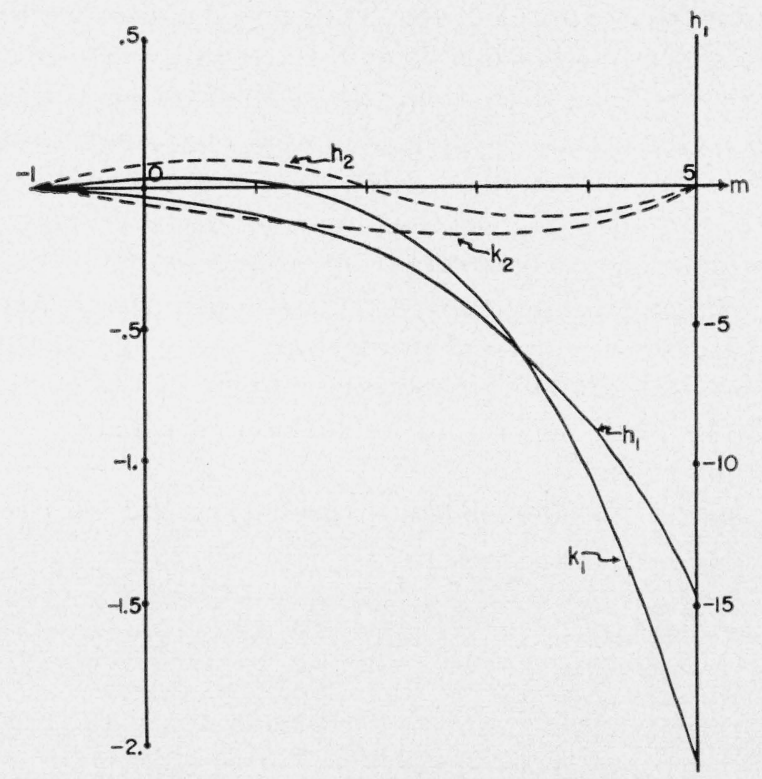

Figure 3 . 
exponential duct causes an antisymmetrical effect on the velocity about the moving midpoint of the flow zone $(m=2)$, whereas the effect on sound speed and density is almost symmetrical about $m=2.8$. On the other hand, in a uniform duct, the resistance effect is a monotonic decrease in the velocity as it approaches the wavefront; it creates a very slight increase in sound speed at the rear of the flow, then a larger decrease in the forward region.

As a numerical example to illustrate the various separate effects, $u, c$, and the local Mach number $M=u / c$ are evaluated in figure $4, \mathrm{~A}$, for $\sigma=1 / 10$; and for $\tau=+1$ in 4 , $\mathrm{B}$, and $\tau=-1$ in 4, C. The dotted lines represent the quantities $u^{0}, c^{0}$, and $M^{0}$ when $\sigma=\tau=0$.

\subsection{An Error Estimate}

As shown by the $c$ curve in figure 4, A, the abscissa of the point where the solid curve intersects the $m$ axis defines the position of the wavefront to the first order approximation. Likewise the corresponding value of $u$ on the curve directly below furnishes an approximation for the velocity of the wavefront. We have, however, already obtained the exact expressions for the position and velocity of the wavefront in (12). The approximations and the full solution agree exactly along line $O B$; the resistive effect is a cumulative one with increasing velocity in the forward part of the wave; hence it is to be expected that the maximum of the approximation error will occur at the wavefront. Thus the error bound obtainable in this way should also be valid for all portions of the wave. Such a calculation for the position of the wavefront indicates that the approximate result will be about 10 percent too large when $\sigma$ grows as large as 0.2 . The other case of variable cross section does not permit this simple method for an error estimate.

\subsection{The Sonic Path}

If $x=s(t, \epsilon)$ is the path along which $u=c$, with $s=0+S \epsilon+0\left(\epsilon^{2}\right)$, the resulting double series expansion correct to first order gives the relation $S(t)=[C(0, t)-U(0, t)] t$. For the case $R=$

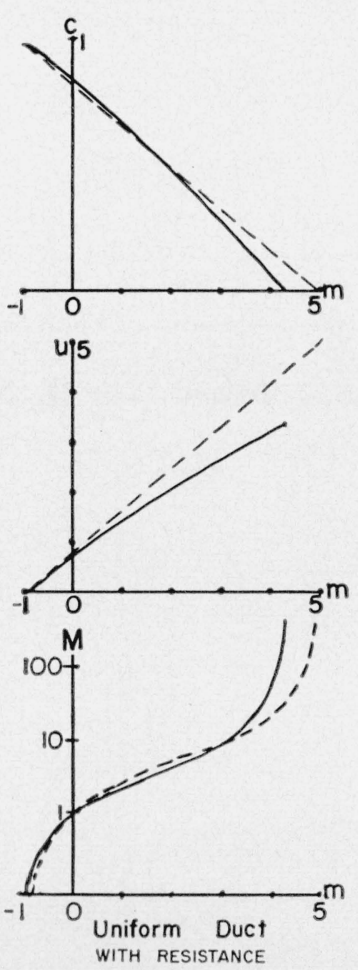

A
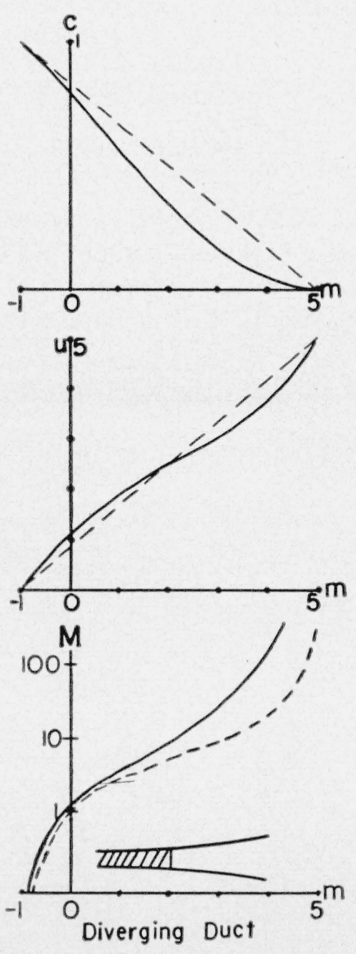

B
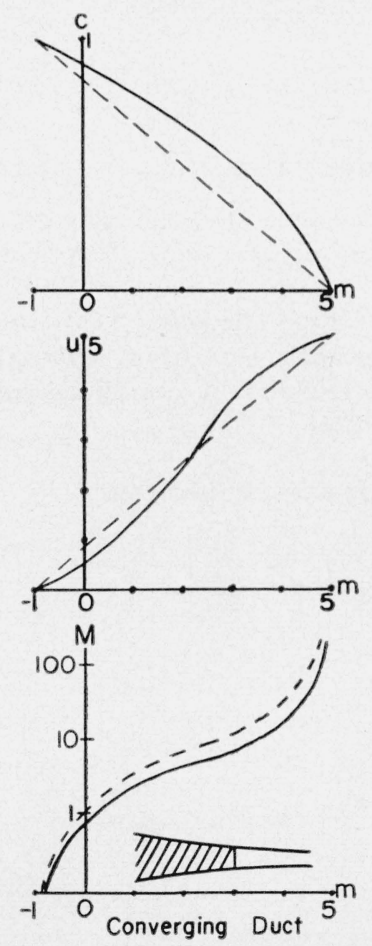

C

Figure 4. 
constant, $a=0$, this defines the sonic path correct to first order to be the downstream parabola

$$
x \doteq 0.23 \epsilon R t^{2} .
$$

For the other case $a=$ constant, $R=0$, we get the parabola

$$
x \doteq-0.18 \epsilon a t^{2},
$$

going upstream or downstream as the duct is diverging or converging, respectively.

\subsection{Flow Past a Fixed Point}

The Mach number distribution across the entire flow at a fixed time has been presented in figure 4. Of more practical interest, however, from the standpoint of applications in shock tubes or wind tunnels is the time variation of local Mach number at a fixed point downstream in the duct, at a point where a fixed model might be placed. This can be derived in an obvious manner from the expressions already presented. As an illustrative example, the Mach number is shown in figure 5 at the fixed point $x=100$ for $\epsilon R=0.005, \tau=0$ in $5, \mathrm{~A}$; for $\epsilon a=+0.01, \sigma=0$ in $5, \mathrm{~B}$; and for $\epsilon a=-0.01, \sigma=0$ in 5 , C. The dotted curve in each figure again represents the $M^{0}$ corresponding to the undisturbed solution.
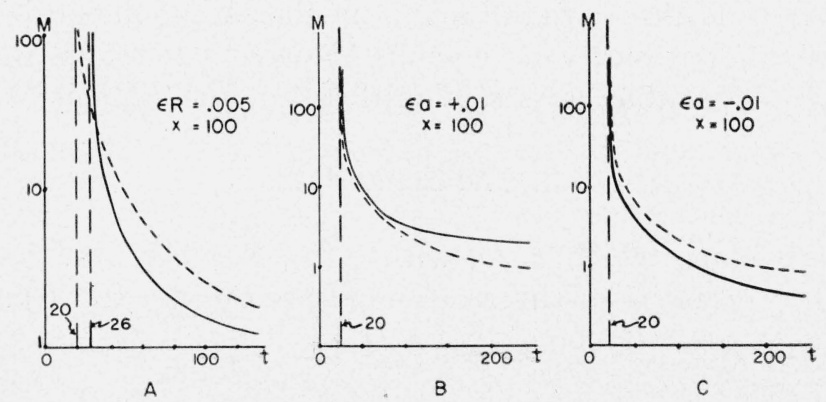

Figure 5.

\section{References}

[1] B. Riemann, Abhandlungen der Gesellschaft der Wissenschaften zu Göttingen, 8 (1860).

[2] R. Courant and K. O. Friedrichs, Supersonic flow and shock waves, Interscience (New York, N. Y., 1948).

[3] R. E. Meyer, On waves of finite amplitude in ducts, Quart. J. Mech. and Appl. Math., vol. V, pt. 3 (1952).

[4] N. A. Hall, The action of friction in nonsteady flow of fluids (First Midwestern Conf. on Fluid Dynamics, Edwards, Ann Arbor, Mich., 1951).

[5] R. F. Dressler, Hydraulic resistance effect upon the dam-break functions, J. Research NBS 22,217 (1952) RP2356.

Washington, February 25, 1954. 